\title{
Estimating preferences for modes of drug administration: The case of US healthcare professionals
}

\author{
Ebenezer K. Tetteh*, Steve Morris, Nigel Titchener-Hooker \\ University College London, UK
}

\begin{abstract}
There are hidden drug administration costs that arise from a mismatch between end-user preferences and how manufacturers choose to formulate their drug products for delivery to patients. The corollary of this is: there are "intangible benefits" from considering end-user preferences in manufacturing patient-friendly medicines. It is important then to have some idea of what pharmaceu-tical manufacturers should consider in making patient-friendly medicines and of the magnitude of the indirect benefits from doing so.

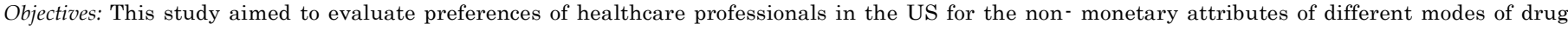
administration. It uses these preference orderings to compute a monetary valuation of the indirect benefits from making patient-friendly medicines.

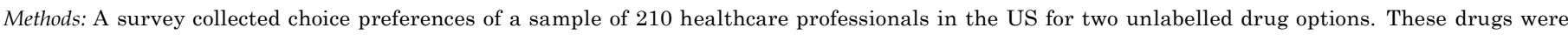

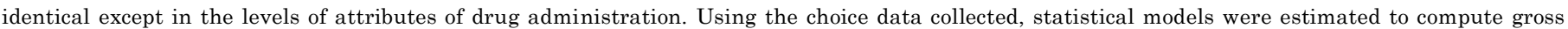
welfare benefits, measured by the expected compensating variation, from making drugs in a more patient-friendly manner.

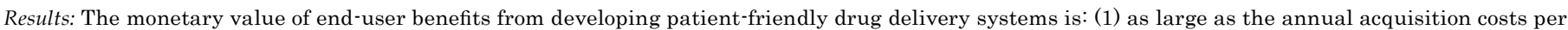
full treatment episode for some biologic drugs; and (2) likely to fall in the "high end" of the distribution of the direct monetary costs of drug administration. Conclusions: An examination of end-user preferences should help manufacturers make more effective and efficient use of limited resources for innovations in drug delivery system, or manufacturing research in general.
\end{abstract}

\section{Introduction}

Different types of resources (pre-treatment counselling and medications; patient education and training for self- administration; medical equipment and consumables; laboratory tests, posttreatment progress checks etc.) are consumed each time a drug is administered. Depending on the type of drug and the disease condition in question, administration of multiple drug doses over time could be a "silent" driver of the direct monetary costs of healthcare delivery. ${ }^{1}$ Granted, a given mode of drug administration that incurs the lowest monetary cost to healthcare payers or providers may incur hidden indirect costs in terms of a

\footnotetext{
* Corresponding author.

E-mail addresses: e.tetteh@alumni.ucl.ac.uk, kwabtetteh@yahoo.com (E.K. Tetteh).
}

mismatch with what is preferred by end-users ${ }^{2} e$ the end-user here being patients or healthcare professionals acting on behalf of patients. Using modes of drug delivery that are out of tune with end-user preferences is thus associated with "intangible costs" that must be accounted for when pharmaceutical manufacturers decide on which production plans to use or research when making clinically-beneficial medicines. The argument here is: if the mode of drug administration is simply a vehicle by which the (incremental) health benefits provided by a drug are delivered to patients, then pharmaceutical manufacturers need to have some knowledge of end-user preferences for this vehicle if they are to produce patient-friendly medicines.

But if patient-friendly medicines are no more than drug products differentiated according to the mode of administration most preferred by end-users, then the obvious question is: whose preferences should be evaluated and taken into account when making these medicines? To answer this question, first 
consider that pharmaceutical R\&D expenditures on medicines (including the costs of manufacturing research) for diseases that are common to both high- and low-income countries are joint global costs to be recouped from all end-users in all countries (submarkets) where a given drug is consumed. ${ }^{3}$ This joint global nature of pharmaceutical $R \& D$ means, in theory, the preferences of all end-users worldwide should be considered, or, at least, endusers in healthcare systems that a manufacturer trades with. Given pharmaceutical $R \& D$ for global diseases is driven largely by economic demand in OECD countries; and the time and re- sources available for this study, we only elicit the preferences of healthcare professionals (doctors and nurses) acting on behalf of patients in the US. We focus on healthcare professionals as they are often responsible for making resource allocation and spending decisions; and because the ultimate end-users ( $\mathrm{pa}^{-}$tients) are usually lessinformed, sometimes passive recipients of medical care.

In this paper, we evaluate healthcare professionals' preferences for the non-monetary characteristics (attributes) of modes of drug administration using a discrete choice experiment (DCE). Our application of a DCE is in accordance with the literature on product variety, notably Spence's ${ }^{4}$ arguments that the most natural way of evaluating the welfare effects of product differentiation is in "attribute space". That is, if end-user preferences for a common set of attributes of a class of products or services is known for a consuming population, then the (expected) demand for or gross consumer welfare benefits gained from any set of products or services that can be described by combinations of these attributes can be estimated. In contrast to working in "attribute space", conventional welfare analysis in "product space", i.e., evaluating demands for products as a whole and not as a combination of attributes, do not allow estimation of demands for hypothetical, non-existent or potential products or services.

\section{Discrete choices and logit demands}

Proposed here is a simplified healthcare market made up of a finite number of pharmaceutical manufacturers on the supply-side and a finite number of end-users: healthcare professionals, acting on behalf of a given patient population, on the demand-side. Each

manufacturer supplies drug products that are identical except for being differentiated according to their mode of administration to patients.

The decision to supply such differentiated drug products is subject to: (1) the resources available for production; (2) the state of underlying manufacturing science; (3) each manufacturer's expectation of incremental private producer surplus from doing so, i.e., the additional revenues net of any additional manufacturing costs; and (4) whether the expected producer surplus covers any additional fixed costs or sunk expenditures on R\&D. The decision to consume these differentiated products is in effect an expression of preferences for a given mode of drug administration. Following characteristics theory, ${ }^{5,6}$ or the "abstract product approach", 7

demand-side utility obtained from each differentiated drug prod- uct is derived not from the drug per se but indirectly from the he- donic characteristics (attributes) of drug administration embodied by that drug.

In this market, demand for a drug product (which is in effect demand for modes of drug administration) can be considered as a derived demand for a bundle of attributes of drug administration. Each drug product can be defined by various possible combinations of attributes and levels (values) for these attributes. These "treatment combinations" of attributes and attribute-levels (or profiles of the products) can be thought of as the output of a transformation matrix that turns attributes into products, and vice versa. Given the prevailing prices of drug products and depending on the resources available to a healthcare professional, i.e., the residual income or financing available after expenditure on a composite of all other healthcare goods and services, one can identify what is called an "attributes efficiency frontier" that indicates the maximum possible combination of attributes and attribute-levels (collection of drug products) that can be afforded.

This, however, assumes that production possibilities allow manufacturers to supply all drug products that all healthcare professionals want or prefer. In the case of limited production possibilities (dictated in part by the state of the underlying manufacturing science), some healthcare professionals may not get what they want or prefer, that is, the product (combination of at- tributes or attribute levels) that maximizes their utility. As a compromise, some healthcare professionals may choose to consume at different times different products for which a combi- nation of profiles of selected products matches their best preferred product if it was supplied by manufacturers. What is clear here is: given limited resources available to manufacturers, and the need to minimize end-user welfare losses, it is crucial that manufacturers have some knowledge of the distribution of healthcare pro- fessionals' preferences in order for them to supply the classes of drug products (differentiated by their mode of administration) that matches closely what the average representative professional rec- ommends or consumes.

Following random utility theory, ${ }^{8}$ the 'satisfaction', 'benefits' or utility $\left(U_{s j}^{*}\right)$ a healthcare professional, $s$, derives from choosing alternative product $j$ from among a set of $J$ differentiated products (which in this case refers to J modes of drug administration) is made of up of two parts. One, a systematic, explainable or observable component, $V_{s j}$ that is a function of the set of attributes; and two, a random unexplained error term, $\varepsilon_{s j}$. We can thus write the following utility function that is linear in parameters and linear in attribute levels:

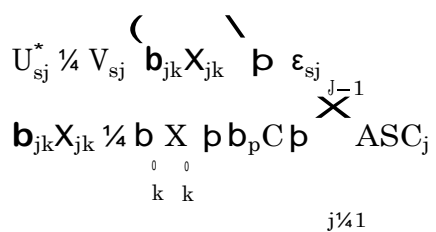

where $X_{j k}$ is a vector of attribute-levels decomposed into $X^{0}, a_{j k}$ vector of generic non-monetary attribute-levels and $C_{p}$, the drug administration cost associated with each alternative product. $b_{j k}$ is a vector of attribute-coefficients, decomposed into $b^{0}, a_{k}$ vector of coefficients for the non-monetary attributes and $b_{p}$, coefficient for the cost attribute. The random error term $\left(\varepsilon_{s j}\right)$ refers to the influence of unobserved or unmeasured factors whilst the alternativespecific-constant $A S C_{j}$ captures any peculiar effects $B_{\text {f }}$ each alternative product that is not reflected in the attributes. $\left({ }_{j=1 / 4}^{\mathbf{J}_{-1} \mathrm{ASC}_{j} \text { may }}\right.$ be considered as the mean of $\varepsilon .{ }_{8} j$

Because each drug product is identical except in the mode of administration, the choice of each healthcare professional is essentially a discrete one. They either chose to have the drug or not: there is no question of how many or how much. Preferences for differentiated drug products can therefore be equivalently described by a distribution of choice probabilities for different modes of drug administration.

Conditional on knowing the vector $b_{j k}$, the probability $(P)$ that $j(1 / 41)$ will be chosen by a given healthcare professional above the other J e 1 discrete products, in each choice situation $(n)$, can be estimated using the "mother" multinomial logit (MNL) model $^{9}$ as follows: 


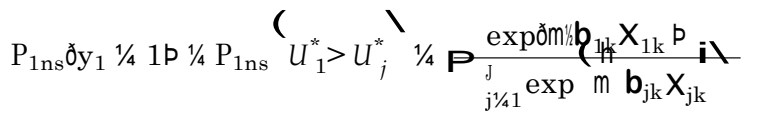

where $y$ denotes the choices made such that $\mathrm{y}_{1}{ }^{1 / 4} 1$ if product $j\left(\begin{array}{ll}1 / 4 & 1\end{array}\right)$ is selected and zero otherwise. Note equation (2) relies on a Gumbel probability density function for an independent and identically distributed (IID) error term, where $\mathrm{m}$ is a positive scale parameter that is inversely related to the error variance. Since the error terms are specific to each choice dataset, $m$ in the "mother" MNL model is usually normalized to one. The normalized IID error terms together with fixed preference coefficients yields the so-called indepen- dence from [ir] relevant alternatives (IIA) assumption e which suggests the ratio of choice probabilities are independent of the inclusion or omission of other products.

One could in a survey or some referendum, collect repeated preferences for products (profiles of attributes and attribute-levels) over a sequence of choice situations e so as to maximize the pool of information that can be collected from a single healthcare professional. The parameters of Equation (2), for the average healthcare professional in the survey sample, can be identified from empirical modelling of the choice data collected.

\section{Methods}

\subsection{Survey development}

The researchers set out to identify a common set of relevant attributes and attribute-levels for different modes of drug administration. This was based on a selective review of Benjamin et al., ${ }^{10}$ Augustovski et al., ${ }^{11}$ Huynh et al., ${ }^{12}$ Parker and Davey, ${ }^{13}$ Dychter et al. ${ }^{14}$; and two systematic reviews. ${ }^{15,16}$ Table 1 below shows our selected set of attributes, definitions of these attributes and their levels. There are three things to note from Table 1.

First, one would preferably want to supplement the literature review above with interviews and/or focus group discussions with members of the sample population of interest. Given the time and resources available for this study, we were not able to apply these qualitative methods $e$ which are of most value where there is a lack

or dearth of existing (grey) literature. We therefore make no claim here that the selected set of attributes and attribute-levels are "exhaustive" of all characteristics of all possible modes of drug administration. We believe, however, that the selected attributes and attribute-levels in Table 1 are relevant and suited for investi- gating the gross welfare benefits from manufacturing patient- friendly medicines. Second, the attribute "risk of non-compli- ance" can be thought of as a composite measure of how a given mode of drug delivery disrupts patients' daily activities; the inci- dence of adverse events specific to the mode of drug delivery (and separate from that of the drug molecule itself) as well as any other factors that might negatively affect treatment compliance, for instance, disutility of pain at the site of drug administration, time and travel costs of accessing healthcare or the absence of insurance cover for medical expenses.

Third, in the analyses, the attribute "dosing frequency" was made into a continuous variable that describes the number of unit administrations of a drug. This was to allow more flexibility in estimating the welfare benefits derived from the decisions and actions taken by healthcare professionals on behalf of patients. For the same reason, the cost attribute was translated into a continuous variable, setting an upper limit of $\$ 20,000$. This is not an arbitrary figure. Our selection of levels for the cost attribute was meant to mimic the empirical distribution of drug administration costs reported elsewhere. ${ }^{17}$ These cost estimates include proximal resource costs incurred before and after physical administration of a drug as well as the costs of physically administering a drug through one of the body's orifices. They exclude drug acquisition costs and other non-drug costs. The maximum cost limit specified is consistent with Farroni et al. ${ }^{18}$ who report that clinical charges for using a room and administering azacitidine in the office (i.e., a clinical

Table 1

Attributes, definitions and attribute levels.

\begin{tabular}{|c|c|c|}
\hline Attributes & Definitions & Levels \\
\hline Method of drug administration & $\begin{array}{l}\text { This attribute refers to the route by which therapeutically-active drug products are } \\
\text { physically administered into a patient. The attribute-levels include "all other needle- } \\
\text { free" methods of drug administration to capture the preferences of patients who desire } \\
\text { oral drug delivery and/or have a fear of needles. }\end{array}$ & $\begin{array}{l}\text { 1. Intravenous delivery } \\
\text { 2. Subcutaneous delivery } \\
\text { 3. Intramuscular delivery } \\
\text { 4. Needle-free delivery }\end{array}$ \\
\hline Dosing frequency & $\begin{array}{l}\text { This attribute refers to the frequency of administering a drug for a single full course of } \\
\text { treatment. Dosing frequency associated with repeated treatments should not be } \\
\text { considered. }\end{array}$ & $\begin{array}{l}\text { 1. Once every six months } \\
\text { 2. Once every month } \\
\text { 3. Once every week } \\
\text { 4. Once every day }\end{array}$ \\
\hline Setting & $\begin{array}{l}\text { This attribute refers to place (clinical and non-clinical settings) where a given drug is } \\
\text { administered. Clinical settings include, for example, hospitals, outpatient clinics, care } \\
\text { homes, offices of general practitioners/physicians etc. Non-clinical settings include } \\
\text { home, schools and other public places. }\end{array}$ & $\begin{array}{l}\text { 1. Clinical } \\
\text { 2. Non-clinical p self-administration } \\
\text { 3. Non-clinical p supervision }\end{array}$ \\
\hline Risk of non-compliance & $\begin{array}{l}\text { This attribute refers to any potential threats to medication compliance or adherence due } \\
\text { to a given mode or method of drug administration and/or recommended dosing } \\
\text { regimen. This is separate from non-compliance due to the safety profile of the drug } \\
\text { molecule. }\end{array}$ & $\begin{array}{l}\text { 1. None } \\
\text { 2. Moderate } \\
\text { 3. Severe }\end{array}$ \\
\hline Risk of medication errors & $\begin{array}{l}\text { This attribute refers to the incidence of common errors of drug administration such as } \\
\text { drug preparation and dosing errors; substitution errors (i.e., giving the wrong drug to } \\
\text { the wrong patient); violation of sterile conditions when drawing up a drug; cuts in glass } \\
\text { ampoules and injection of minute shards of glass with the drug etc. }\end{array}$ & $\begin{array}{l}\text { 1. None } \\
\text { 2. Moderate } \\
\text { 3. Severe }\end{array}$ \\
\hline Cost & $\begin{array}{l}\text { This attribute refers to the additional resource costs (per patient per full treatment } \\
\text { course) incurred in administering drugs to patients. }\end{array}$ & $\begin{array}{l}\text { 1. } \$ 200 \\
\text { 2. } \$ 1000 \\
\text { 3. } \$ 3000 \\
\text { 4. Over } \$ 3000\end{array}$ \\
\hline
\end{tabular}

Notes:

a This refers to the situation where people, if properly trained, could self-administer the drug in a non-clinical setting; or otherwise, their medications will have to be

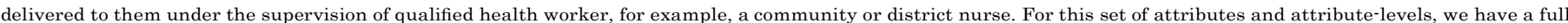
factorial of $1728\left(1 / 44^{3} 3^{3}\right)$ possible profiles or treatment combinations. 
setting) ranges from $\$ 300-\$ 500$ per injection: charges per patient per year for a full treatment course of six cycles ranges from $\$ 12,600-\$ 21,000$. We believe $\$ 20,000$ is a reasonable upper limit since charges do not always match costs.

Having identified the most relevant attributes and attributelevels, the investigators developed an "efficiency choice" experimental designs that form the basis of our DCE survey questionnaires. The experimental design embodies the transformation matrix for turning products into bundles of attributes and bundle of attributes into products. For this survey, we decided to present participants with the same sequence of choice situations, where in each situation a respondent had to choose between two unlabelled drugs $A$ and $B$ that are identical in every aspect apart from the manner in which they are administered to patient. A forced-choice format was employed by not offering a constant "none" or "opt- out" alternative. This is because of the difficulty in imagining that healthcare professionals will deny or withhold a clinically benefi- cial drug simply because the way in which the drug is administered is not what they or their patients prefer.

The investigators developed basic experimental designs, estimating the main effects of the attribute-levels. This was done in SAS v. $9.3^{19,20}$, resulting in a 24 choice-set experimental design that had a relative D-efficiency of $52.24 \%$. This design was chosen based on joint considerations of its relative $\mathrm{D}$-efficiency and its statistical properties when merged with an artificial pre-pilot dataset created with simulated choice data. Compared to other experimental de-

signs, this design yielded the lowest standard errors over all attribute-coefficients estimated using the same "mother" MNL model with the same simulated discrete-choice data. The in-

vestigators considered that a 24 -choice-set design might impose greater cognitive burden (task complexity) for survey respondents, leading perhaps to irrational or inconsistent choices. ${ }^{21}$ This issue was resolved by blocking this design into two versions $e$ each version consisting of 12 randomly assigned choice sets. No prior information on attribute effects was used in developing the designs.

\subsection{Survey administration}

The survey questionnaires, developed from the blocked experimental designs above, were split into three sections. The first section of each questionnaire provided a preamble with informa- tion about the purpose of the study and the hypothetical "con- structed" context in which respondents have to make their choices. It also provided descriptions of the attributes and attribute-levels as well as an example of a completed choice set as a guide for the survey respondents. Respondents were asked to make their choices assuming they were "fund holders", i.e., they had financial responsibility over the allocation and use of resources for healthcare provision. The second section contained the actual sequence of 12 choice questions or situations. And, the third section collected anonymized information on individual respondents' characteristics.

A small-scale informal pilot of the questionnaires with no more than five people was used to test the wording of the questionnaire; to ensure that the instructions were clear and to identify what might be perceived as implausible combination of attributes and attributelevels. We found that it took, on average, $15 \mathrm{e} 20 \mathrm{~min}$ to complete each block questionnaire. Following the pilot phase, small wording changes to the questionnaires were made to improve clarity. In line with recommendations made by the ISPOR Good Research Practices for Conjoint Analysis Task Force, ${ }^{22}$ a sample size of survey participants was chosen that exhausted the resources available for this study. With the help of a commercial vendor (Survey Monkey), the questionnaires were administered online to the target sample of US doctors and nurses. It took roughly two weeks for the vendor to complete the surveys. Our dataset provided 5040 usable choice responses from 210 survey respondents. The vendor provided no information on number of people they contacted in order to achieve the minimum number of respondents, and for that reason we cannot compute a survey response rate.

Given that online panels of survey respondents could vary from actual populations of interest, it cannot be said that the sample is representative of all healthcare professionals in the US. Demographic characteristics of the estimation sample are shown in Table 2 below.

\subsection{Statistical modelling}

To adequately capture variations in choice data, a number of analyses were conducted, starting with the "mother" MNL model, i.e., Equation (2). The second model estimated is the heteroskedastic multinomial (HMNL) model, where the scale parameter is no longer normalized to one but considered a variable that must be estimated. In this case, the error terms are longer IID distributed. With the scale parameter expressed as a function of a vector of respondents' characteristics (Z), the probability of an individual respondent choosing alternative product $j$ from among the set of competing products in a given choice situation is given by:

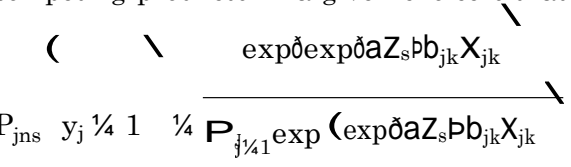

where $\mathrm{a}$ is a vector of parameters reflecting the influence of respondents' characteristics on the error variance. If $k$ is not statistically different from zero, the HMNL model closely approximates

Table 2

Demographic characteristics of sample.

\begin{tabular}{|c|c|}
\hline Characteristics (SN) & $\mathrm{N}$ (\% of sample) \\
\hline \multicolumn{2}{|l|}{ Gender (209) } \\
\hline Male $^{\mathrm{a}}$ & $65(31.10)$ \\
\hline Female & $144(68.90)$ \\
\hline \multicolumn{2}{|l|}{ Respondents' age (208) } \\
\hline Under 50 years & $105(50.48)$ \\
\hline$:: 50$ years & $103(49.52)$ \\
\hline \multicolumn{2}{|c|}{ Type of healthcare institution (209) } \\
\hline Solo medical practice & $14(6.70)$ \\
\hline Government-run & $15(7.18)$ \\
\hline Private-for-profit & $67(32.06)$ \\
\hline Non-for-profit & $89(42.58)$ \\
\hline Other $^{\mathrm{a}}$ & $24(11.48)$ \\
\hline \multicolumn{2}{|l|}{ Patient case-mix (209) } \\
\hline Inpatients & $78(37.32)$ \\
\hline Outpatients & $80(38.28)$ \\
\hline Accidents \& emergency & $22(10.53)$ \\
\hline Other $^{\mathrm{a}}$ & $29(13.88)$ \\
\hline \multicolumn{2}{|l|}{ US census region (206) } \\
\hline East North Central & $31(15.05)$ \\
\hline East South Central & $10(4.85)$ \\
\hline Middle Atlantic & $27(13.11)$ \\
\hline South Atlantic ${ }^{\mathrm{a}}$ & $38(18.45)$ \\
\hline Mountain & $16(7.77)$ \\
\hline New England & $22(10.68)$ \\
\hline Pacific & $24(11.65)$ \\
\hline West North Central & $20(9.71)$ \\
\hline West South Central & $18(8.74)$ \\
\hline
\end{tabular}

Notes: N 1/4 number of respondents.

a Indicates the reference category for the effects-coding used (see also Table 3). Our dataset offered a complete sequence of 12 choices for each of the 210 survey respondents. However, for three respondents, we had missing data on some of their characteristics whilst for one respondent we had no information on all characteristics. 
the "mother" MNL. ${ }^{21}$

The third model is the entropy multinomial logit (EMNL) in which the scale parameter is a function of entropy $(E)$ : a measure of the variation represented in the probability distribution of a discrete random variable, in this case the choice variable $y$. In DCE literature, entropy summarizes the impact of respondent fatigue. The argument is: even if survey respondents are identical, differ-

ential amounts of effort exerted in making choices will appear as unobserved heterogeneity in preferences. Here we express the

scale parameter as a function of entropy of each choice situation:

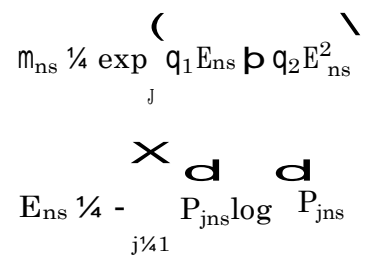

where $\boldsymbol{F}_{\text {jns }}$ is the estimated choice probability from the "mother" MNL model; $q_{1} ; q_{2}$ are parameters associated with entropy. The quadratic function for the scale parameter is intended to estimate non-linearity in unobserved heterogeneity captured by the mea-

sure of entropy above. The case of $\mathcal{G}_{1}<0$ and $\mathcal{q}_{2}>0$ indicates respondent fatigue, i.e., no learning effects and declining effort as respondents work through a sequence of choice sets.

The fourth is a mixed multinomial logit (MMNL) model in which $b$ varies randomly across individual respondents. In this case, the IIA restriction no longer holds albeit the error terms remain IID. A common practice is to assume the individual-specific preference Table 3

Table 3
Explanatory variables.

\begin{tabular}{|c|c|}
\hline Variables & Definitions (Effects coding) \\
\hline INTRAVENOUS & $1 / 41$ if a drug is administered intravenously $(1,0,0,-1)$ \\
\hline SUBCUTANEOUS & $1 / 41$ if a drug is administered subcutaneously $(0,1,0,-1)$ \\
\hline INTRAMUSCULAR & $1 / 41$ if a drug is administered intramuscularly $(0,0,1,-1)$. The reference category $(-1)$ is administration via needle-free routes \\
\hline DOSFREQ & $\begin{array}{l}\text { This refers to the number of unit administrations for a single full course of treatment over a one year period. The variable allows the attribute- } \\
\text { levels: "once every day", "once every week", "once every month" and "once every six months" to be expressed on a continuous quantitative scale }\end{array}$ \\
\hline NONCLINICAL_SELF & $1 / 41$ if a drug is self-administered in non-clinical settings $(1,0,-1)$ \\
\hline NONCLINICAL_SUPV & $\begin{array}{l}1 / 41 \text { if a drug is administered in non-clinical settings under the supervision of a qualified healthcare professional, zero otherwise }(0,1,-1) \text {. The } \\
\text { reference category }(-1) \text { is drug administration in clinical settings }\end{array}$ \\
\hline NONCOMP_MODERATE & $1 / 41$ if the risk of patient non-compliance associated with a given mode of drug administration is moderate $(1,0,-1)$ \\
\hline NONCOMP_SEVERE & $\begin{array}{l}1 / 41 \text { if the risk of patient non-compliance associated with a given mode or method of drug administration is severe }(0,1,-1) \text {. The reference } \\
\text { category }(-1) \text { is drug delivery that is associated with no risk of non-compliance }\end{array}$ \\
\hline RME_MODERATE & $1 / 41$ if the risk of medication errors made by health staff or by patients self-administering is moderate $(1,0,-1)$ \\
\hline RME_SEVERE & $\begin{array}{l}1 / 41 \text { if the risk of medication errors made by health staff or by patients self-administering is severe }(0,1,-1) \text {. The reference category }(-1) \text { is drug } \\
\text { delivery that is associated with no risk of medication errors }\end{array}$ \\
\hline COST & $\begin{array}{l}\text { This refers to the cost of resources expended on drug administration per patient per single full treatment course over a year. It excludes drug } \\
\text { acquisition costs }\end{array}$ \\
\hline A & Alternative-specific constant for the drug $A$ option $(1,-1)$. The reference point, drug option $B \frac{1}{4}-1$ \\
\hline FEMALE & $1 / 41$ if survey respondent is female $(1,-1)$. The reference category $(-1)$ are males \\
\hline RESPONDENTAGE & Continuous variable indicating the age of a survey respondent \\
\hline SOLO & $1 / 41$ if a respondent works in a solo medical practice $(1,0,0,0,-1)$ \\
\hline GOVERNMENT & $1 / 41$ if a respondent works in a government-run healthcare institution $(0,1,0,0,-1)$ \\
\hline PRIVATE-FOR-PROFIT & $1 / 41$ if a respondent works in a private-for-profit healthcare institution $(0,0,1,0,-1)$ \\
\hline NON-FOR-PROFIT & $\begin{array}{l}1 / 41 \text { if a respondent works in a non-for-profit healthcare institution }(0,0,0,1,-1) \text {. The reference category }(-1) \text { are respondents who work in all } \\
\text { other healthcare institutions not mentioned above }\end{array}$ \\
\hline INPATIENTS & $1 / 41$ if a respondent caters to inpatients $(1,0,0,-1)$ \\
\hline OUTPATIENTS & $1 / 41$ if a respondent caters to out-patients $(0,1,0,-1)$ \\
\hline EMERGENCY & $\begin{array}{l}1 / 41 \text { if a respondent caters to patients in accident and emergency departments }(0,0,1,-1) \text {. The reference category }(-1) \text { are respondents who cater } \\
\text { to all other kinds of patients }\end{array}$ \\
\hline ENC & $1 / 41$ if a respondent's healthcare institution is located in the East North Central census region $(1,0,0,0,0,0,0,0,-1)$ \\
\hline ESC & $1 / 41$ if a respondent's healthcare institution is located in the East South Central census region $(0,1,0,0,0,0,0,0,-1)$ \\
\hline MA & $1 / 41$ if a respondent's healthcare institution is located in the Middle Atlantic census region $(0,0,1,0,0,0,0,0,0,-1)$ \\
\hline
\end{tabular}

where $R$ is the number of Halton simulations; and $b_{s j k}$ is the $r$ th draw from $f$ ðbjథp. ${ }^{25}$

The fifth is a latent-class multinomial logit (LCMNL) model that assumes preference coefficients are drawn from a mixture of nonparametric discrete distributions, representing $C$ latent classes of pomogenous subpopulations. It is not known a priori which latent

class an individual belongs to. So unconditional on class membership, the choice probability $\left(P^{*}\right)$ in a given choice situation is estimated as:

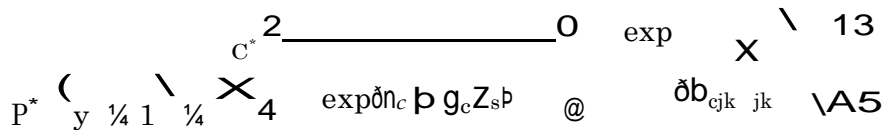

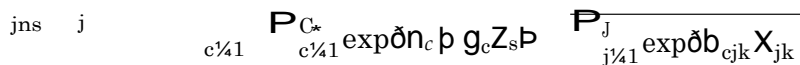

where the vector $g \delta 1 / 4 g_{1} ; g_{2} ; \ldots ; g_{C} P$ refers to the effect of 
$1 / 41$ if a respondent's healthcare institution is located in the Mountain census region $(0,0,0,1,0,0,0,0,-1)$

$1 / 41$ if a respondent's healthcare institution is located in the New England census region $(0,0,0,0,0,1,0,0,0,-1)$

$1 / 41$ if a respondent's healthcare institution is located in the Pacific census region $(0,0,0,0,0,0,1,0,0,-1)$

$1 / 41$ if a respondent's healthcare institution is located in the West North Central census region $(0,0,0,0,0,0,0,1,0,-1)$

WNC

$1 / 41$ if a respondent's healthcare institution is located in the West South Central census region $(0,0,0,0,0,0,0,0,1,-1)$. The reference category

$(-1)$ are respondents who work in healthcare institutions located in the South Atlantic census region

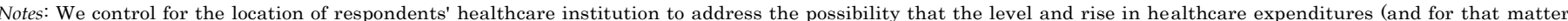

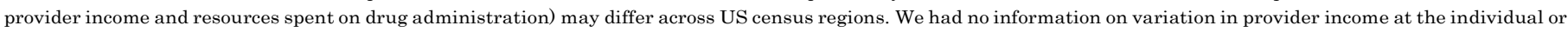
institutional level. 
respondents' characteristics on $\mathrm{p}_{c}$, the probability of class mem bership $\left({ }_{C^{1} / p_{c} 1 / 41}\right)$; and $n_{c}$ is a vector of class-specific constants. $C^{*}$ is op number of latent-classes that yields the lowest timal

consistent Akaike Information criterion (cAIC) and/or the lowest Bayesian Information Criterion (BIC). Here we determined $C^{*}$ by estimating two to ten LCMNL models. ${ }^{26}$

The models above were estimated in STATA v. 11 using the effects-coded explanatory variables in Table 3 below. Variables in the shaded portion of Table 3 are those related to respondents' characteristics, i.e., the $\mathbf{Z}$ variables in Equation (6).

\subsection{Measuring end-user benefits}

As a measure of welfare benefits, the marginal willingness-topay (MWTP) was calculated for a single non-cost attribute under consideration e assuming there is only one product available that will be chosen with a $100 \%$ certainty. MWTP is ratio of coefficients for that attribute to the coefficient for the cost attribute. Classical confidence intervals for the MWTP estimates were generated using 100 bootstrap replicates. That is, a sampling distribution of MWTP estimates using 100 bootstrap resamples was created from the original dataset. Each bootstrap resample was created by randomly drawing individual observations with replacement whilst ensuring each resample matches the structure of the original choice data, i.e., the individual observations are (1) clustered according to indicators for respondents and (2) grouped, within each cluster, according to indicators for the choice sets. Of course, a higher number of replicates is needed for more precise estimation but we prefer this procedure as it is: (1) computationally less demanding; (2) uses actual data from respondents without making parametric assumptions about the distribution of MWTP; and (3) compatible with all STATA estimators for the HMNL, EMNL, MMNL and LCMNL models.

The expected compensating variation (ECV), which is a more valid measure of benefits when there is uncertainty about which product will be chosen, was also calculated For discrete-choice probabilities estimated using an MNL-type model:

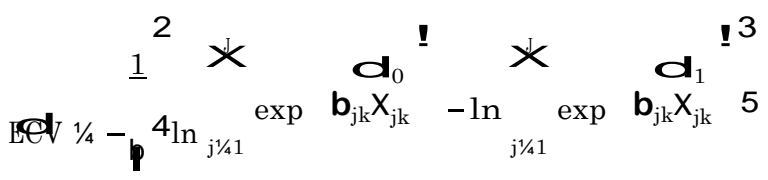

where $\mathbf{I}$ is the marginal utility of income proxied by the negative coefficient of the price/cost attribute; and the superscripts 0 and 1 denote what was available before and after a product switch. ECV measures the difference in gross welfare benefits in moving from the initial (0) to the final (1) state ${ }^{27}$ For an example of valuing "product innovations" using this ECV metric, see Trajtenberg's ${ }^{28}$ work on computed axial tomography scanners. In this paper, "product innovation" refers to different modes of drug administration e and our ECV estimates provide a single-period monetary value of what might be considered "intangible benefits" of giving endusers what they want in terms of the mode of drug delivery. The intertemporal flows of benefits are not considered: ECV esti- mates are not expressed in discounted present values. Classical confidence intervals for the ECV estimates were generated using 100 bootstrap replicates.

\section{Results}

Table 4 above shows the results from our analyses. Based on the log-likelihoods and AIC, the LCMNL model with two classes offers the best fit to our choice data. Further support for the LCMNL model is provided by similarity with results for the MMNL model. That said the HMNL and EMNL models provide additional insights as to the nature of the choices observed.

First, note that the coefficients of the HMNL model are quite similar to that of the MNL model whilst a Lagrangian Multiplier test similar to that of the MNL model whilst a Lagrangian Multiplier test for heteroskedasticity indicated statistically insignificant unobserved heterogeneity ( $p$ value 10.1064 ). This preference certainty or minimal residual unobserved variance appears to be explained by whether US healthcare professionals work in private-for-profit or not-for-profit healthcare institutions, and whether they cater for inpatient healthcare demands. It also appears to be explained by whether a given healthcare professional works in the MiddleAtlantic census region as opposed to the South-Atlantic census region (which is the reference category).

Second, note the loss of statistical significance of the attributecoefficients obtained from the EMNL model. We know from equation (4) that the EMNL model estimates a quadratic relationship between the scale parameter and a measure of entropy (which indicates the degree of randomness or "unlikeliness" of the choice dataset). It appears that, in the presence of statistically insignificant unobserved heterogeneity (identified from the HMNL model), the EMNL model fails to identify appreciable entropy within the choice data. Consequently, the (unrestricted) EMNL model does not significantly fit the data better than the (restricted) MNL model. The additional explanatory power provided by the EMNL model over and above the MNL model is small. What is more the statistically insignificant entropy parameters $\left(\mathscr{G}_{1} ; q_{2}\right)$ mean we cannot reject arguments that there are no significantrespondent-fatigue effects. Still the magnitude and signs of the insignificant entropy parameters suggests initial exertion of effort is offset later by an equivalent decline in effort. To add, the MNL and HMNL models indicate a statistically significant alternative-specific-constant: a preference for option $B$ (listed on the right side of each choice set in the survey) as opposed to option $A$ (listed on the left). We can therefore say respondents switch from full evaluation of the attributes at the beginning of the DCE questionnaire to decisionsimplifying heuristics based on partial information towards the end.

Third, the improvement in log-likelihood observed for the MMNL model (relative to the MNL model) confirms there are, at least, some significant variations in and correlations between the

preference coefficients we specified ex ante as random. However, these variations and correlations in preferences can be equivalently captured by the LCMNL model without making parametric assumptions about their distributions. In fact, the LCMNL model we estimated captures correlations between preference coefficients

that vary across respondents whilst in the MMNL model, these correlations are constant across respondents unless one links parameters of the continuous mixture distribution to the $Z$ variables.

Focusing on the LCMNL model, we observed that latent-class membership is dictated by whether respondents cater to inpatient healthcare demands: the variable INPATIENTS was the only statistically significant predictor of latent-class membership. Gender, age (a proxy for years of experience), institutional type and

US census region all had no statistically significant effect on class membership. Conditional on membership of latent-class 1 , the average or representative respondent was indifferent to the choice

between intravenous and needle-free modes of drug administration. (Holding all else equal, the coefficient for the INTRAVENOUS

variable was not statistically significant.) The results suggested a positive preference for subcutaneous modes of drug administration and a negative preference for intramuscular drug delivery $e$ albeit the latter is only statistically significant at the $10 \%$ level.

The investigators observed a negative preference for drug delivery modes that involve high dosing frequency although the magnitude of this effect is small. Note also the positive preference for self-administration by patients in non-clinical settings. On the 


\begin{tabular}{|c|c|c|c|c|c|c|}
\hline \multicolumn{7}{|c|}{ Dependent variable: CHOICE PROBABILITY } \\
\hline \multirow[t]{3}{*}{ Variables/Coefficients: } & \multirow{3}{*}{$\begin{array}{l}\text { MNL model } \\
\text { bóSEP }\end{array}$} & \multirow{3}{*}{$\begin{array}{l}\text { HMNL model } \\
\text { B(SE) }\end{array}$} & \multirow{3}{*}{$\begin{array}{l}\text { EMNL model } \\
\text { BöSEP }\end{array}$} & \multirow{3}{*}{$\begin{array}{l}\text { MMNL model } \\
F_{\mathrm{s}} 0 \mathrm{SEP}\end{array}$} & \multicolumn{2}{|l|}{ LCMNL model } \\
\hline & & & & & $\mathrm{b}_{1} \mathrm{DSEP}$ & $\mathrm{b}_{2}$ ðSEP \\
\hline & & & & & $\mathrm{b}$ & \\
\hline INTRAVENOUS & $0.261(0.291)$ & $0.146(0.217)$ & $0.129(0.151)$ & $0.149(0.351)$ & $0.343(0.386)$ & $1.612(1.046)$ \\
\hline SUBCUTANEOUS & $0.519(0.103)^{k+k x}$ & $0.401(0.103)^{k+k * k}$ & $0.222(0.244)$ & $0.453(0.120)^{\text {*k* }}$ & 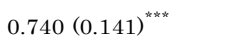 & $0.125(0.354)$ \\
\hline INTRAMUSCULAR & $-0.111(0.105)$ & $-0.063(0.079)$ & $-0.053(0.059)$ & $-0.200(0.124)$ & $-0.259(0.145) !$ & $-0.231(0.376)$ \\
\hline DOSFREQ & $-0.003(0.000)^{* * * *}$ & $-0.002(0.000)^{* * * k}$ & $-0.001(0.001)$ & $-0.003(0.000)^{k+2 * k}$ & $-0.004(0.000)^{* * * k}$ & $-0.002(0.001)$ \\
\hline NONCLINICAL_SELF & $0.442(0.056)^{k+k+k}$ & $0.322(0.073)^{k+2 \times k}$ & $0.197(0.183)$ & $0.481(0.060)^{k, k * k}$ & $0.513(0.067)^{k+\frac{k}{2}}$ & $0.429(0.189)^{*}$ \\
\hline NONCLINICAL_SUPV & $-0.097(0.050) !$ & $-0.059(0.039)$ & $-0.043(0.045)$ & $-0.127(0.056)^{*}$ & $-0.060(0.063)$ & $-0.439(0.170)^{* *}$ \\
\hline NONCOMP_MODERATE & $-0.070(0.050)$ & $-0.044(0.039)$ & $-0.021(0.037)$ & $-0.143(0.064)^{*}$ & $-0.077(0.067)$ & $-0.111(0.131)$ \\
\hline NONCOMP_SEVERE & $-0.200(0.045)^{k * * k}$ & $-0.149(0.043)^{* \ldots k}$ & $-0.083(0.091)$ & $-0.120(0.052)^{*}$ & $0.337(0.065)^{k . k \times k}$ & $0.205(0.143)$ \\
\hline RME_MODERATE & $0.006(0.052)$ & $-0.003(0.040)$ & $0.006(0.022)$ & $0.042(0.071)$ & $-0.170(0.072)^{*}$ & $0.521(0.143)^{* * \hbar k}$ \\
\hline RME_SEVERE & $-0.339(0.041)^{k * * * \hbar}$ & $-0.260(0.061)^{k+k \cdot k}$ & $-0.145(0.155)$ & $-0.445(0.046)^{*+k}$ & $-0.075(0.052)$ & $-1.528(0.206)^{* . * *}$ \\
\hline COST & $-0.00006(0.000)^{k * * *}$ & $-0.00004(0.000)^{* k * k}$ & $-0.00002(0.000)$ & $-0.0006(0.0028)^{*}$ & $-0.00006(0.000)^{* * * *}$ & $-0.00005(0.000)^{k * * *}$ \\
\hline A & $-0.329(0.092)^{* * * *}$ & $-0.236(0.080)^{* * \hbar}$ & $-0.146(0.145)$ & $-0.323(0.108)^{* . *}$ & $-0.418(0.124)^{k+k+k}$ & $-0.696(0.322)^{*}$ \\
\hline 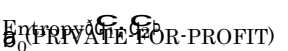 & $\stackrel{e}{e}$ & $\stackrel{e}{0.210(0.098)^{*}}$ & $\stackrel{(3.748,-3.781)}{e}$ & $\stackrel{e}{e}$ & $\stackrel{e}{e}$ & \\
\hline 最(INPA-FOR-PROFIT) & e & $0.26991^{\left.(0.100)_{2}\right)^{* *}}$ & - & è & 巳 & \\
\hline \multicolumn{7}{|l|}{ b } \\
\hline \multicolumn{7}{|l|}{$b$} \\
\hline $\mathrm{a}_{3}(\mathrm{MA})$ & e & $0.254(0.125)^{k}$ & e & e & e & \\
\hline $\mathbf{p}_{c}$ & e & e & e & e & 0.72 & 0.28 \\
\hline $\mathrm{g}_{c}$ (INPATIENTS) & e & e & e & e & $0.820(0.360)^{*}$ & \\
\hline $\mathrm{LL}^{0}$ & -1746.731 & -1446.647 & -1446.647 & -1446.647 & & \\
\hline $\mathrm{LL}^{\mathrm{b}}$ & -1446.647 & -1416.264 & $-1445.726 y$ & -1399.013 & -1325.1643 & \\
\hline $\mathrm{AIC}$ & 2917.293 & 2888.527 & 2919.453 & 2852.026 & 2732.328 & \\
\hline
\end{tabular}

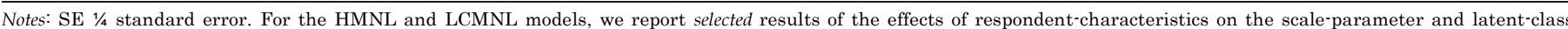

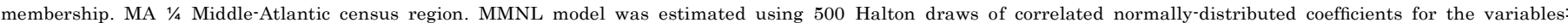

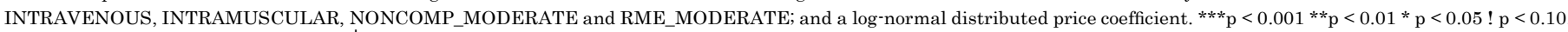

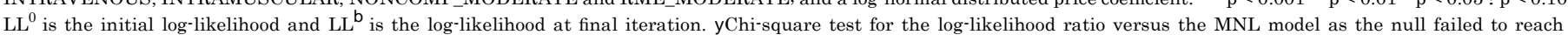
statistical significance ( $p$ vale $1 / 4$ 0.3984). AIC 1/4 Akaike Information Criterion.

other hand, the average respondent was indifferent to drug administration in non-clinical settings under the supervision of a qualified healthcare professional. This offers further support to the notion that if administering a drug in a non-clinical setting requires supervision by a qualified healthcare professional, then one might be better off administering that drug in a clinical setting. The average respondent was willing to accommodate drug delivery methods that are associated with a "moderate" risk of treatment non-compliance (in that the coefficient for the variable for mod- erate risk of treatment non-compliance was not statistically sig- nificant) but exhibited a negative preference for drug delivery modes that carry a "severe" risk of patient non-compliance. That we observed a negative preference for even "moderate" risks of medication errors possibly reflects concerns about patient safety, professional reputation and medical malpractice suits. Although it is not statistically significant, we observed a negative preference for severe risk of medication errors.

The results for latent-class 2 differ in the following ways. The variables SUBCUTANEOUS, INTRAMUSCULAR and DOSFREQ all have statistically insignificant effects. The variable for severe risk of treatment non-compliance also had no statistically significant effects. We observed, however, a statistically significant negative preference for drug administration in non-clinical settings under the supervision of a qualified healthcare professional. The results indicate a positive (less risk-averse) preference for modes of drug delivery that are associated with "moderate" risk of medication errors but a significant negative preference for those that carry a "severe" risk of medication errors.

It is worth mentioning that the MNL model is equivalent to an LCMNL model with one homogenous latent class. Hence, given our LCMNL model with two classes show there is an over $70 \%$ probability of a respondent belonging to latent-class 1, we should expect close similarity between the MNL and LCMNL models. Indeed, the BIC for the MNL model (2995.595) is slightly lower than that for the LCMNL model (2999.467) e although the latter offers a substantial improvement in the log-likelihoods. Given the statistically insignificant unobserved heterogeneity identified by the HMNL and EMNL models, we can say this choice dataset is one instance where estimating an MNL model without consideration of alternative models might not lead to grossly misleading conclusions.

Table 5 shows MWTP estimates for the non-cost attributes studied and the associated $95 \%$ confidence intervals. As expected, we observe differences in the sign and magnitude of MWTP from the different models. Focusing on the LCMNL model, these figures indicate a high MWTP for intravenous and subcutaneous modes of drug delivery compared to needle-free routes of drug administra- tion e plus a high MWTP to avoid intramuscular modes of drug administration. Observe also the high MWTP for selfadministration in non-clinical settings and a high MWTP to avoid drug delivery modes that are associated with a "severe" risk of treatment non-compliance and/or "severe" risk of medication errors.

Next evaluated is the welfare change from reverse engineering a given formulation of drug $C$ to a more patient-friendly version $D$. If we assume that both versions of the drug have the same molecule, efficacy and safety profile. Drug $C$ is manufactured for intravenous administration in clinical settings, and this mode of drug delivery is associated with "severe" risk of treatment non-compliance and "severe" risk of medication errors. Drug $D$ is manufactured for subcutaneous self-administration in non-clinical settings and this mode of drug delivery is associated with "moderate" risk of 
Table 5

Marginal willingness-to-pay estimates (in \$1000).

\begin{tabular}{|c|c|c|c|c|c|}
\hline \multirow[t]{2}{*}{ Variables: } & \multirow{2}{*}{$\frac{\text { MNL model }}{\text { M }}$} & \multirow{2}{*}{$\frac{\text { HMNL model }}{\text { MWP ð95\% CIP }}$} & \multirow{2}{*}{$\frac{\text { EMNL model }}{\text { M }}$} & \multirow{2}{*}{$\frac{\text { MMNL model }}{\text { M }}$} & \multirow{2}{*}{$\begin{array}{l}\text { LCMNL model } \\
\text { MГРð95\% CIP }\end{array}$} \\
\hline & & & & & \\
\hline INTRAVENOUS & $4.56(3.69,5.44)^{\mathrm{a}}$ & $2.53(1.58,3.47)^{\mathrm{a}}$ & $6.34(5.04,7.63)^{\mathrm{a}}$ & $2.17(0.95,3.38)^{\mathrm{a}}$ & $10.67(8.53,12.82)^{\mathrm{a}}$ \\
\hline SUBCUTANEOUS & $9.33(8.92,9.73)^{a}$ & $9.90(9.55,10.27)^{\mathrm{a}}$ & $9.44(8.98,9.91)^{\mathrm{a}}$ & $7.290(6.83,7.75)^{\mathrm{a}}$ & $10.61(0.71,11.51)^{\mathrm{a}}$ \\
\hline INTRAMUSCULAR & $-1.91(-2.26,-1.56)^{\mathrm{a}}$ & $-1.14(-1.49,-0.08)^{\mathrm{a}}$ & $-2.49(-2.99,-1.99)^{\mathrm{a}}$ & $-4.708(-5.20,-4.21)^{\mathrm{a}}$ & $-4.13(-5.00,-3.27)^{\mathrm{a}}$ \\
\hline DOSFREQ & $-0.049(-0.051,-0.048)^{\mathrm{a}}$ & $-0.051(-0.053,-0.05)^{a}$ & $-0.051(-0.052,-0.049)^{\mathrm{a}}$ & $-0.048(-0.050,-0.047)^{\mathrm{a}}$ & $-0.058(-0.062,-0.055)^{\mathrm{a}}$ \\
\hline NONCLINICAL_SELF & $7.82(7.59,8.04)^{\mathrm{a}}$ & $7.80(7.61,8.00)^{\mathrm{a}}$ & $9.02(8.60,9.44)^{\mathrm{a}}$ & $9.08(8.88,9.29)^{\mathrm{a}}$ & $8.21(7.66,8.77)^{\mathrm{a}}$ \\
\hline NONCLINICAL_SUPV & $-1.60(-1.76,-1.43)^{\mathrm{a}}$ & $-1.34(-1.50,-1.19)^{\mathrm{a}}$ & $-1.68(-1.88,-1.47)^{\mathrm{a}}$ & $-2.70(-2.88,-2.52)^{\mathrm{a}}$ & $-1.91(-2.21,-1.615)^{\mathrm{a}}$ \\
\hline NONCOMP_MODERATE & $-1.27(-1.43,-1.11)^{\mathrm{a}}$ & $-1.00(-1.172,-0.83)^{\mathrm{a}}$ & $-0.66(-0.89,-0.44)^{\mathrm{a}}$ & $-3.53(-3.78,-3.28)^{\mathrm{a}}$ & $-1.71(-2.03,-1.40)^{\mathrm{a}}$ \\
\hline NONCOMP_SEVERE & $-3.58(-3.72,-3.44)^{\mathrm{a}}$ & $-3.53(-3.66,-3.39)^{\mathrm{a}}$ & $-3.55(-3.73,-3.36)^{\mathrm{a}}$ & $-1.42(-1.61,-1.23)^{\mathrm{a}}$ & $-3.74(-4.04,-3.44)^{\mathrm{a}}$ \\
\hline RME_MODERATE & $0.04(-0.11,0.19)$ & $-0.21(-0.38,-0.03)^{\mathrm{a}}$ & $0.50(0.27,0.73)^{\mathrm{a}}$ & $1.05(0.67,1.44)^{\mathrm{a}}$ & $-0.16(-0.47,0.15)$ \\
\hline RME_SEVERE & $-5.98(-6.24,-5.72)^{\mathrm{a}}$ & $-6.22(-6.45,-5.98)^{\mathrm{a}}$ & $-6.10(-6.43,-5.78)^{\mathrm{a}}$ & $-8.35(-8.60,-8.10)^{\mathrm{a}}$ & $-7.59(-8.14,-7.03)^{\mathrm{a}}$ \\
\hline
\end{tabular}

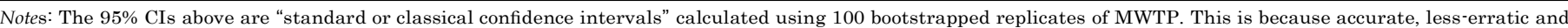

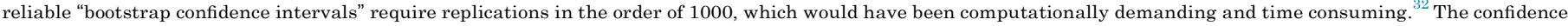

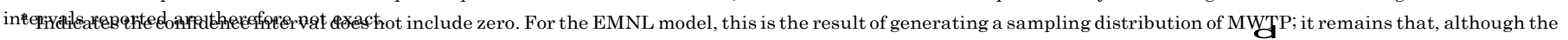

model parameters are identified, the EMNL model offers no significant improvement in log-likelihood over the MNL model.

treatment non-compliance and "moderate" risk of medication errors. Based on the MNL model, switching from drug $C$ to $D$ yields an ECV (per patient per single full treatment course over a year) of $-\$ 22,790$ (95\% CI: $-\$ 23,562$ to $-\$ 22,018)$. Based on the MMNL model, the ECV is $-\$ 31,601$ (95\% CI: $-\$ 32,374$ to $-\$ 30,828) .{ }^{1}$ Based on the LCMNL model with two latent classes, and unconditional on class membership, LCV is - $\$ 24,932$ (95\% CI: - $\$ 26,653$ to - $\$ 23,212)$.

Note that the negative sign before the ECV estimates simply reflects Equation (7); ECV is a measure of welfare gain, not a loss in benefits.

Fig. 1 shows the kernel density plots of the distribution of ECV derived from the MNL, MMNL and LCMNL models. Notice, the considerable overlap of the plots for the models; in particular the closer overlap between the kernel density plots for the LCMNL and MNL models. This provides some reassurance as to the accuracy of the welfare estimates obtained. The difference between the plots for the LCMNL and MMNL models probably reflects the different assumptions about preference heterogeneity. We believe that the reason why we see a wider spread of the kernel density plot of ECV derived from the LCMNL model (relative to the MNL or MMNL models) is: because the LCMNL estimates are based on the average coefficients over the two-latent classes (with weights given by the probability of latent-class membership). Hence, if the probabilities of class-membership do not remain constant or fixed for each of the 100 bootstrap resamples, one would obtain a lot more variation in ECV than if (fortuitously) the proportion of individuals belonging to each of the latent classes does not vary from one bootstrap resample to the other. That the plot for the MNL model overlaps

\footnotetext{
${ }^{1}$ A retransformation problem that plagues estimation of MMNL models with lognormal coefficients. The cost coefficient reported in Table 4 for the MMNL model $\left(\mathscr{F}_{\mathrm{p}} \mathrm{p}\right.$ was derived from the distribution of its logged form, $\ln \varnothing \mathrm{b}_{\mathrm{p}} \mathrm{P}$, usifg the following retransformation: $b_{\mathrm{p}}{ }^{1 / 4}$ expðlnð $b_{\mathrm{p}} \mathrm{pp} 0: 5 \mathrm{SD}^{2} \mathrm{P}_{\text {where }} \mathrm{SD}^{1 / 4}$ standard de- viation for $\ln \mathrm{b}_{\mathrm{p}}$ $\mathrm{P}$ : This, howevE, assumes the individual $\ln \mathrm{b}_{\mathrm{p}} \mathrm{P}$ recovered frofm the dataset are normally-distributed with a constant variance. If this is not the case, one obtains a biased and less consistent retransformed cost coefficient. Whilst the retransformed cost coefficient may bracket that obtained MMNL models with fixed or normally-distributed cost coefficients, this may introduce significant differences in subsequent welfare analysis. Here in computing the welfare change of switching from option $C$ to $D$, we used the cost coefficient $\left(1 \boldsymbol{K}_{\mathrm{p}} 1 / 4\right.$ 0.000066) from an unreported MMNL model (with a lower log-likelihood $1 / 4-1427.922$ and specified with a normally-distributed cost coefficient) as this figure is comparable with cost co efficients from the other models. We consider this a reasonable solution to the retransformation problem as the other non-cost coefficients from the unreported MMNL model are similar to the MMNL model reported in Table 4. The problem is obviously situation specific and we suggest further discussions and research on how best to resolve it.
}

more with that of the LCMNL model confirms that, for this dataset, welfare estimates based on the MNL model are less biased.

\section{Discussion}

The ECV estimates, which provide a monetary value of the welfare gain from making patient-friendly medicines (1) are in the same order of magnitude as the annual acquisition costs $(\$ 17,017$ to $\$ 41,888$ ) for some biologic drugs ${ }^{29}$; and (2) exceed the annual direct monetary costs of administering most drugs (for a single full treatment course). They are likely to fall in the "high end" of the distribution of the direct monetary costs of drug administration reported elsewhere. ${ }^{17}$

The results above suggest a strong positive preference for modes of drug administration that are associated with some but not significant risks of treatment non-compliance and/or medication errors. Relative to needle-free routes, there is either a positive preference or indifference to intravenous and subcutaneous drug delivery; plus a somewhat negative preference for or indifference to intramuscular modes of administration. The results also show a consistent preference for self-administration of drugs in non- clinical settings $e$ and a consistent negative preference for drug administration in clinical settings or non-clinical settings under the supervision of a qualified healthcare professional. For this reason, we will encourage advances in pharmaceutical manufacturing such as "closed vial access devices" or "closed-system transfer devices" that allows healthcare professionals to safely reconstitute and administer what might be considered hazardous drugs.

Granted, it might be argued that the sign and magnitude of MWTP from the LCMNL model for the INTRAVENOUS and SUBCUTANEOUS variables is counterintuitive as one would expect a positive preference for less-invasive needle-free routes. However, there are sound clinical reasons for a positive MWTP for intravenous and subcutaneous delivery, relative to needle-free routes (even if the effect is not always statistically significant). Not all drugs, especially in emergency situations, can be given via needle- free routes; and the pharmacokinetic profile of some drugs (for example, those that have a narrow therapeutic index) may be such that intravenous administration is the only or most appropriate route of drug delivery. Considering also the variable for re- spondents who cater for inpatient populations is the only statisti- cally significant predictor of latent-class membership (institutional context and all other $\mathbf{Z}$ variables in Table 3 had no statistically significant effects on latent-class membership), the positive $\mathrm{co}^{-}$efficients for the INTRAVENOUS and SUBCUTANEOUS variables are 


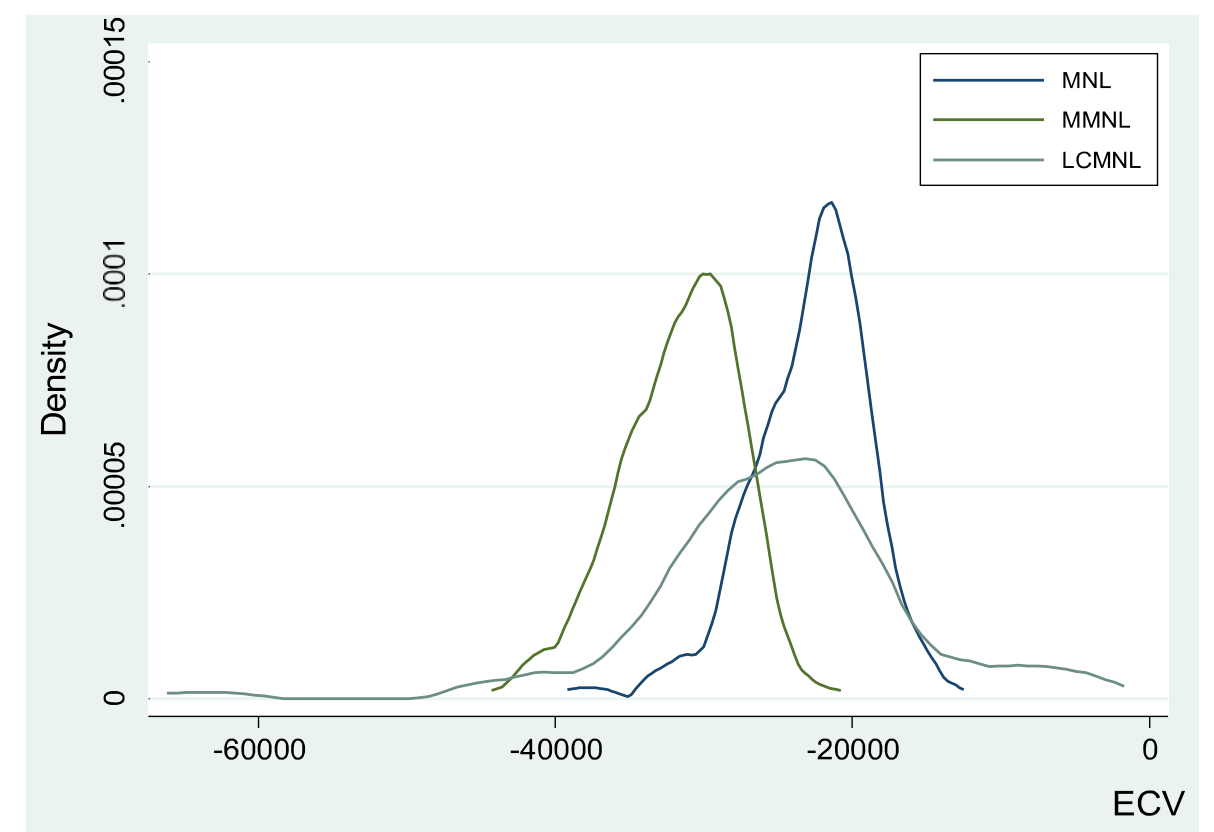

Notes: $\widehat{\mathrm{ECV}}$ refers to the welfare change of switching from drug option $C$ to $D$. The plots are based on 100 bootstrapped estimates of ECV. This is because estimation of more accurate confidence intervals (that require replications in the order of 1000), would have been computationally demanding and time consuming.

$\mathrm{MNL}=$ mulitnomial logistic model; $\mathrm{MMNL}=$ mixed multinomial logistic model; $\mathrm{LCMNL}=$ Latent class multinomial model

Fig. 1. Sample distribution of expected compensating variation estimates.

not as surprising as it first seems. In fact, this is consistent with a DCE of French physicians' preferences for intravenous and oral cancer chemotherapy that showed a positive preference for intravenous administration in curative settings as opposed to a positive preference for oral (needle-free) administration in palliative noncurative settings. ${ }^{10}$

Further, it might be argued that MP and LCV derived from the LCMNL model suffers from ecological fallacy e as they are based on the average weighted coefficients over two latent-classes. For that matter (erroneous) conclusions that apply at the aggregate level may not apply at the latent-class level. However, we do not know a priori which latent-class a given respondent belongs to $e$ and we cannot assume fixed class membership. Hence the esti- mates reported here, which are unconditional on class member- ship, are a valid measure of welfare benefits.

Notwithstanding, a number of limitations apply here. First, in an attempt to capture the global nature of the pharmaceutical industry, this research only evaluated the preferences of US healthcare professionals at a given point in time. Some sort of temporal averaging of stated choices across independently drawn sample populations at different time points may help reduce any timedependent measurement noise that casts doubts about the external validity of the findings reported here. That said, the gen- eral question of whether preferences for modes of drug adminis- tration differ from country to country is an empirical matter that requires an extensive multicountry DCE beyond that presented in this paper.

Second, the hypothetical choice scenarios presented to US healthcare professionals required that they act as fund-holders taking on payer responsibilities. It might then be argued that the choice data collected may be different from what might have been elicited from actual healthcare payers (who are also agents acting on behalf of patients). On the other hand, payers in their managerial accounting roles rarely administer drugs to patients themselves: they are less familiar with the day-to-day clinical needs of patients. Also, payers are more likely to concern themselves with the direct costs and direct health benefits a drug offers rather than the attributes of drug administration and the associated intangible benefits. On balance, asking healthcare professionals to make discrete drug choices assuming they had financial control of healthcare resources is a better approach compared to a sample population of payers e not to mention the practical problems of identifying such a sample. Note, however, that the choice data collected was not in a form that allows subgroup analyses of the preferences of doctors and nurses. That the preferences of doctors differ from that of nurses might be worth pursuing in a future DCE study designed to investigate this issue. Related to this is the observation that imperfect agency means the choices of healthcare professionals may not necessarily match what patients want or prefer. The DCE results, however, do not require healthcare professionals to act as perfect agents on behalf of patients. The negative coefficients for the attributes "severe risk of medication errors" and "severe risk of treatment compliance" indicates that although healthcare professionals may be risk-averse and focus on their own utility, patients' well-being are not ignored. Patients, especially less- informed passive ones, indirectly obtain the gross welfare bene- fits measured in this paper via the decisions and actions taken by healthcare professionals on their behalf.

Third is the possibility that using categorical variables for the cost attribute may lead to different results or, at least, reveal nonlinearities in the price elasticity of "demands" for the non- monetary attributes studied. For the choice dataset, however, 
even if such non-linearities exits, the near-zero coefficients for the cost attribute is such that this is unlikely to significantly change the arguments and conclusions reached.

Bar these limitations, it is clear that the gross (supposedly intangible) welfare benefits gained from patient-friendly modes of drug administration are not trivial. Payers, providers and other agents in healthcare markets therefore need to recognize not just the value of the drug molecule (i.e., the net health benefits it offers) but also value of the drug delivery mode or method. But there is evidence that the mode of drug delivery could also improve health outcomes, i.e., amplify the magnitude of direct health benefits derived from the drug molecule or perhaps reduce the uncertainty around real-life experience of these health benefits. Delea et al., ${ }^{30}$ for instance, show that changing drug delivery systems may not only reduce the monetary costs of drug administration but also confer health benefits expressed in terms of quality-adjusted life- years (QALYs) to patients. These potential increments in QALYs (measuring direct health benefits) are separate from the ECV estimates that measure indirect end-user benefits.

Admittedly, on the supply-side, the expected additional revenues net of manufacturing costs (private producer surplus) and the state of manufacturing and formulation science must be adequate and capable enough to support the desired innovations and efforts to make patient-friendly medicines. The value created via product differentiation will then depend on whether a manufacturer is able to reorganize its technical capabilities to pursue a successful "cost strategy", i.e., reducing manufacturing costs over time; or a successful "benefits strategy", i.e., increasing value through larger increases in gross end-user benefits; or both. ${ }^{31}$ The point here is: if the discounted present value of private producer surplus of such value-creation initiatives (relative to other investment opportunities) is positive, then there is no reason why manufacturers should not consider the switch from drug option $C$ to $D$. The incentive for a manufacturer to develop patient-friendly drug delivery systems, however, exists only if healthcare payers/providers are willing to pay for the value of the drug delivery mode, i.e., they are willing to channel prescribing and consumption choices (shift market shares) towards products with the preferred attributes of drug administration. In which case, effective and efficient use of pharmaceutical manufacturing research resources will require, at least, knowledge of what attributes end-users want most and what combination of attributes offer the maximum possible (change in) welfare benefits, which in turn will offer the largest (change in) welfare benefits net of any schedule of (changes in) manufacturing costs.

\section{Conclusion}

This study found a non-trivial marginal willingness-to-pay for drug delivery systems that are associated with zero or moderate risk of medication errors and/or treatment non-compliance. It also found a high marginal willingness-to-pay for self-administration of drugs in non-clinical settings. In addition, it was estimated that the monetary value of making patient-friendly medicines is as large as the annual acquisition costs of some biologic drugs and/or are likely to fall in the "high-end" of the distribution of the direct monetary costs of drug administration. The discrete choice experiment provides additional insights as to what biopharmaceutical manufacturers should pay attention to in making patient-friendly medicines. An examination of end-user preferences should help manufacturers make more effective and efficient use of limited resources for innovations in drug delivery systems, or manufacturing research, in general.

\section{References}

1. Tetteh E, Morris S. Systematic review of drug administration costs and implications for biopharmaceutical manufacturing. Appl Health Econ Health Policy. 2013:1e12.

2. Chess R. Economics of drug delivery. Pharm Res. 1998;15:172e174.

3. Tetteh EK. Implementing differential pricing for essential medicines via country-specific bilateral negotiated discounts. Appl Health Econ Health Policy. 2009; $7: 71$ e89.

4. Spence M. Product differentiation and welfare. Am Econ Rev. 1976:407e414.

5. Lancaster KJ. A new approach to consumer theory. I Polit Econ. 1966;74: $132 \mathrm{e} 157$.

6. Lancaster K. Socially optimal product differentiation. Am Econ Rev. 1975: $567 e 585$.

7. Baumol WJ. Calculation of optimal product and retailer characteristics: the abstract product approach. J Polit Econ. 1967:674e685.

8. Manski CF. The structure of random utility models. Theory Decis. 1977;8: $229 \mathrm{e} 254$.

9. McFadden D. Conditional logit analysis of qualitative choice behavior. In: Zarembka P, ed. Frontiers in Econometrics. New York: Academic Press; 1974: $105 e 142$.

10. Benjamin L. Physicians' preferences for prescribing oral and intravenous anticancer drugs: a discrete choice experiment. Eur J Cancer. 2012;48:912.

11. Augustovski F, Beratarrechea A, Irazola V, et al. Patient preferences for biologic agents in rheumatoid arthritis: a discrete-choice experiment, Value Health. 2013;16:385e393.

12. Huynh TK, Oÿstergaard A, Egsmose C, Madsen OR. Preferences of patients and health professionals for route and frequency of administration of biologic agents in the treatment of rheumatoid arthritis. Patient Prefer Adherence. 2014;8:93

13. Parker SE. Pharmacoeconomics of intravenous drug administration. Pharmacoeconomics. 1992;1:103e115.

14. Dychter SS. Subcutaneous drug delivery: a route to increased safety, patient satisfaction, and reduced costs. J Infusion Nurs. 2012;35:154.

15. McDowell SE, Mt-Isa S, Ashby D, Ferner RE. Where errors occur in the preparation and administration of intravenous medicines: a systematic review and Bayesian analysis. Qual Saf Health Care. 2010;19:341e345.

16. Keers RN, Williams SD, Cooke J, Ashcroft DM. Prevalence and nature of medication administration errors in health care settings: a systematic review of direct observational evidence. Ann Pharmacother. 2013;47:237e256.

17. Tetteh E, Morris S. Evaluating the administration costs of biologic drugs: development of a cost algorithm. Health Econ Rev. 2014;4:26.

18. Farroni JS, Zwelling L, Cortes J, Kantarjian H. Saving medicare through patientcentered changes - the case of injectables. N Engl J Med. 2013;368:1572e1573.

19. Kuhfeld WF, Tobias RD. Large factorial designs for product engineering and marketing research applications. Technometrics. 2005:47.

20. Kuhfeld WF. Marketing Research Methods in SAS Experimental Design, Choice, Conjoint, and Graphical Techniques. 2009.

21. Louviere JJ, Islam T, Wasi N, Street D, Burgess L, Designing discrete choice experiments: do optimal designs come at a price? J Consumer Res. 2008;35: $360 \mathrm{e} 375$.

22. Bridges JFP. Conjoint analysis applications in health - a checklist: a report of the ISPOR good research practices for conjoint analysis task Force. Value health. 2011;14:403.

23. Swait J. Choice environment, market complexity, and consumer behavior: a theoretical and empirical approach for incorporating decision complexity into models of consumer choice. Organ Behav Hum Decis Process. 2001;86:141.

24. de Bekker-Grob EW, Rose JM, Bliemer MC. A closer look at decision and analyst error by including nonlinearities in discrete choice models: implications on willingness-to-pay estimates derived from discrete choice data in healthcare. PharmacoEconomics. 2013;31:1169e1183.

25. Hole AR. Estimating mixed logit models using maximum simulated likelihood. Stata I. 2007:7:388e401.

26. Pacifico D. Fitting nonparametric mixed logit models via expectationmaximization algorithm. Stata J. 2012:12.

27. Lancsar E, Louviere J. Conducting discrete choice experiments to inform healthcare decision making. Pharmacoeconomics. 2008;26:661e677.

28. Trajtenberg M. The welfare analysis of product innovations, with an application to computed tomography scanners. J Polit Econ. 1989;97:444e479.

29. Wu N, Shah N, Harrison DJ. Cost of biologics per treated patient across immune-mediated inflammatory disease indications in a pharmacy benefit management setting: a retrospective cohort study. Clin Ther. 2014;36: $1231 e 1241$.

30. Delea TE, Sofrygin O, Thomas SK, Baladi JF, Phatak PD, Coates TD. Cost effectiveness of once-daily oral chelation therapy with deferasirox versus infusional deferoxamine in transfusion-dependent thalassaemia patients: US healthcare system perspective. Pharmacoeconomics. 2007;25:329e342.

31. Besanko D, Gupta S, Jain D. Logit demand estimation under competitive pricing behavior: an equilibrium framework. Manag Sci. 1998;44:1533e1547.

32. Efron B. Better bootstrap confidence intervals. J Am Stat Assoc. 1987;82: 171e185. 